\title{
Splashing of a very viscous liquid drop impacting onto a solid wall wetted by another liquid
}

\author{
Hannah M. Kittel*, Ilia V. Roisman, Cameron Tropea \\ Institute for Fluid Mechanics and Aerodynamic, Technische Universität Darmstadt, Germany \\ *Corresponding author: kittel@sla.tu-darmstadt.de
}

\begin{abstract}
In this experimental work the main focus is on the impact of a single drop of a very viscous liquid onto a thin, horizontal wall film of different liquid. Splashing resulting from drop impact onto a wetted wall occurs in many natural and engineering applications like in internal combustion engines and spray cooling. While the splashing threshold for low viscosity liquid drops has been extensively examined, impact of a very viscous drop is much less studied. The viscosities of drop and wall film liquids are varied up to kinematic viscosities of $100,000 \mathrm{~mm}^{2} / \mathrm{s}$. The liquids used in the experiments are miscible.

The impact outcome is determined by the impact parameters and fluid properties. The effect of very viscous liquids used as drop fluid and as wall film liquid on the kinematic of the corona expansion is investigated in the experiments. The results of drop impact onto solid walls are compared to obtain the limiting asymptotic values for the splashing threshold.

Finally, a semi-empirical model for the splashing threshold, for the maximum spreading radius $D_{\max }$ and for the maximum spreading times $t_{\max }$ are developed for extremely viscous liquids.
\end{abstract}

\section{Keywords}

Drop impact, high viscous liquids, wetted wall

\section{Introduction}

Splashing as a result of a drop impact onto a thin liquid film [1-3] or onto a dry substrate [4-6] occurs in many natural and engineering applications like spray coating, spray cooling, paint spraying, ink-jet printing and in internal combustion engines. Impact of a supercooled drop onto a surface of an aircraft can lead to ice accretion, while collision with a heated substrate can lead to drop evaporation or intensive boiling [7]. The drop/wall interaction is affected by the fact that the drop and the liquid film are different liquids and may exhibit different degrees of miscibility and viscosity. A comprehensive review on the phenomena, modeling and application of drop impact can be found in [8]. Drop impact onto a wetted wall can lead to various different outcomes. In the case of splashing
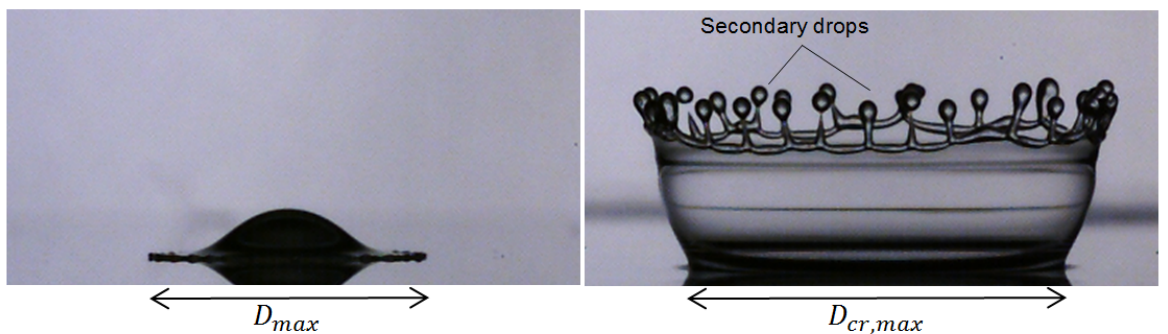

Figure 1. Different impact outcomes, on the left deposition, on the right corona splash.

a radial expanding flow in the lamella is formed after the drop impact. Due to the interaction with the outer liquid wall film an uprising liquid sheet is generated which is bounded by a Taylor rim [10-12]. If the rim gets unstable, finger-like jets are formed which lead to the generation of secondary drops [14]. In this case the inertial effects in the spreading lamella dominate over the surface tension and viscosity effects, and if the crown jets are unsteady, secondary droplets are formed. It is well-known that the splashing threshold of a drop impacting onto a wetted wall is determined by the inertia, viscous and capillary forces. If no instabilities occur or the instabilities do not lead to secondary drops, the phenomena is called corona. If the drop spreads on the impact surface, it is called deposition. In this case the spreading diameter is determined by the flow in the lamella and the propagation of the rim [9]. The dimensionless Weber and Reynolds number as well as the substrate wettability determine furthermore the maximum spreading diameter. If the drop rebounds completely or partially, it is called rebound or partial rebound. The impact outcome is highly determined by the impact parameters, like the impact velocity $u_{0}$ and the initial drop diameter $d_{0}$, and in the case of different liquids by the fluid properties, like the kinematic viscosity $\nu$ and the surface tension $\sigma$. To describe these phenomena the relative film thickness $\delta=h / d_{0}$ and the viscosity ratio $\nu_{0}=\nu_{f} / \nu_{d}$ are 
introduced. In order to define a splashing threshold, whether splashing occurs or not, the dimensionless $\mathrm{K}$ number is introduced [13]. A critical value is determined that defines the splashing threshold. The splashing threshold is dependent on the impact velocity as well as on the viscosity of both liquids of drop and wall film. While the splashing threshold of low viscosity liquid drops has been extensively examined in the past [13], impact of a very viscous drop is much less studied, despite its relevance to such intensively developing fields like additive manufacturing.

The main subject of this study is the experimental investigation of the effect of very high viscous liquids on the outcome of drop impact. The maximum spreading diameter $D_{\max }$ in the case of deposition and the maximum corona diameter $D_{c r, \max }$ in the case of corona/corona splash as well as the maximum spreading time $t_{\max }$ are determined for various fluid combinations. The splashing/deposition limit is determined using the modified $\mathrm{K}^{*}$ number as a function of the viscosity ratio $\nu_{0}$.

\section{Material and methods}

The experimental setup is shown schematically in Fig. 2 and consists of three main parts, the droplet generating unit, the impact substrate and the observation system. In order to generate a single droplet, the micro pump transports the liquid from a tank to the cannula. At the end of the tip the droplet is collected until it reaches a critical mass and drips off. Since the droplet dripping is driven by gravity the impact velocity is determined by the distance of the tip of the cannula and the impact substrate. In this study the impact velocity is set to $u=3.2 \mathrm{~m} / \mathrm{s}$. In order to vary the initial droplet diameter $d_{0}$ different cannula diameter are used. As impact substrate a horizontal glass plate is used. In order to generate a wall film the glass plate is sandblasted up to a recess. A ring with a diameter of $60 \mathrm{~mm}$ is glued on this sandblasted area. The unsealed area is used to create the wall film. To measure the film thickness a micrometer screw is used. The tip of the screw is first lowered till it touches the glass substrate to determine the reference value. After the liquid is filled in the screw is lowered till it reaches the liquid surface. The difference of both values determines the film thickness.

The impact substrate is located between the observation system. The observation system consists of a highspeed camera (Photron FASTCAM SA-X2) and a light-emitting diode (LED, Veritas Constellation 120E) with 12,000 Lumen. A diffusing plate is placed in front of the LED in order to receive a consistent light. In this study a frame rate of $30,000 \mathrm{fps}$ is used with a resolution of $896 \times 480$ pixel. The shutter time is set to $12.5 \mu \mathrm{s}$. The physical properties of the liquids which are used in this study can be found in Table 1. The viscosities of drop and wall film liquids are varied up to kinematic viscosities of $100,000 \mathrm{~mm}^{2} / \mathrm{s}$. The liquids used in these experiments are miscible.

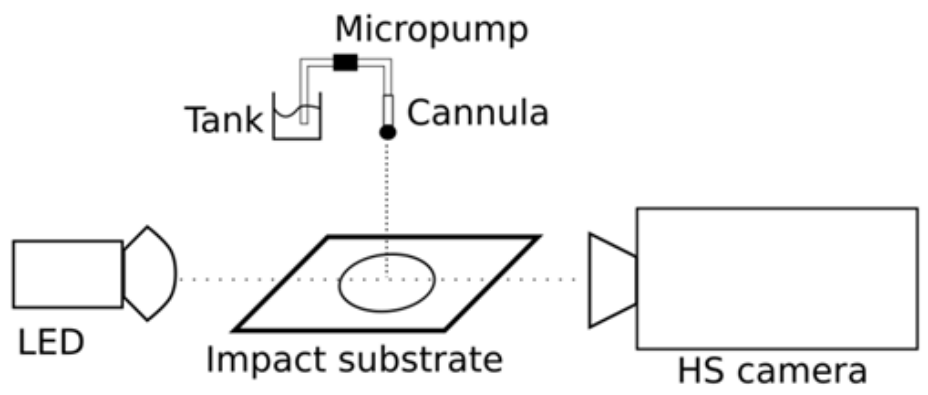

Figure 2. Schematic illustration of the experimental setup

Table 1. Physical properties of the liquids used in this study.

\begin{tabular}{c|ccc}
\hline & Kinematic viscosity $\left[\mathrm{mm}^{2} / \mathrm{s}\right]$ & Surface tension $[\mathrm{mN} / \mathrm{m}]$ & Density $\left[\mathrm{kg} / \mathrm{m}^{3}\right]$ \\
\hline H2O & 1.004 & 72.24 & 997 \\
Hexadecane & 4.11 & 27.61 & 769.15 \\
S5 & 5 & 17.72 & 910 \\
S10 & 10 & 18.19 & 920 \\
S20 & 20 & 18.2 & 925 \\
S65 & 65 & 18.2 & 950 \\
S350 & 350 & 18.56 & 972 \\
S500 & 500 & 18.83 & 972 \\
S750 & 750 & 18.6 & 972 \\
S1000 & 1,000 & 18.59 & 972 \\
S10000 & 10,000 & 18.81 & 972 \\
S30000 & 30,000 & 18.81 & 972 \\
S100000 & 100,000 & 18.81 & 972
\end{tabular}




\section{Results and discussion}

In order to see if there is a connection between the outcome of drop impact onto a solid wall and onto a high viscous liquid film the viscosity of the wall film is varied up to $100,000 \mathrm{~mm}^{2} / \mathrm{s}$. A solid surface can be considered as a substrate with infinite viscosity.

The impact of a drop leads to different outcomes dependent on the impact substrate. If the impact substrate is covered by a very high viscosity liquid or is a solid surface the impact results in deposition, as it can be seen in Fig. 3. The maximum spreading diameter $D_{\max }$ as well as the maximum spreading time $t_{\max }$ are mainly determined by the viscosity of the drop and the impact substrate. In this case the viscosity of the wall film determines the phenomena itself, deposition, but the viscosity of the drop determines the geometrical and temporal parameters like $D_{\max }$ and $t_{\max }$. Comparing the outcomes of the same drop impacting onto different wall film liquids, as it can be seen in Fig. 3, the outcome does not differ much from each other, whereby comparing the outcomes of the same wall film liquid but different drop liquids the outcomes differ significantly from each other. The impact substrate determines the motion of the drop after the impact, whether the drop can build a corona or the fluid of the drop is stopped and end in deposition. The higher viscous the film gets the less motion of the drop is enabled due to the viscous forces. In the case of deposition the higher viscous the drop gets the less spreading occurs also due to the viscous forces. The motion of the drop is stopped due to the inaction of the drop. Directly after the drop impact a very high viscous drop only sits on the impact substrates, but does not spread what is caused by the high viscosity and its loss of ambition to change its shape. In the first phase of a couple milli seconds a very high viscous drop gets only compressed breadth wise, then the motion of the drop is stopped. In the second phase which lasts much longer, several seconds, the contact angle decreases slowly. The viscosity of the liquids highly influences the impact outcome due to its characteristic of motion depending on its magnitude.

If the liquid of the impact substrate is less viscous the drop impact leads to the evolution of a corona. Furthermore
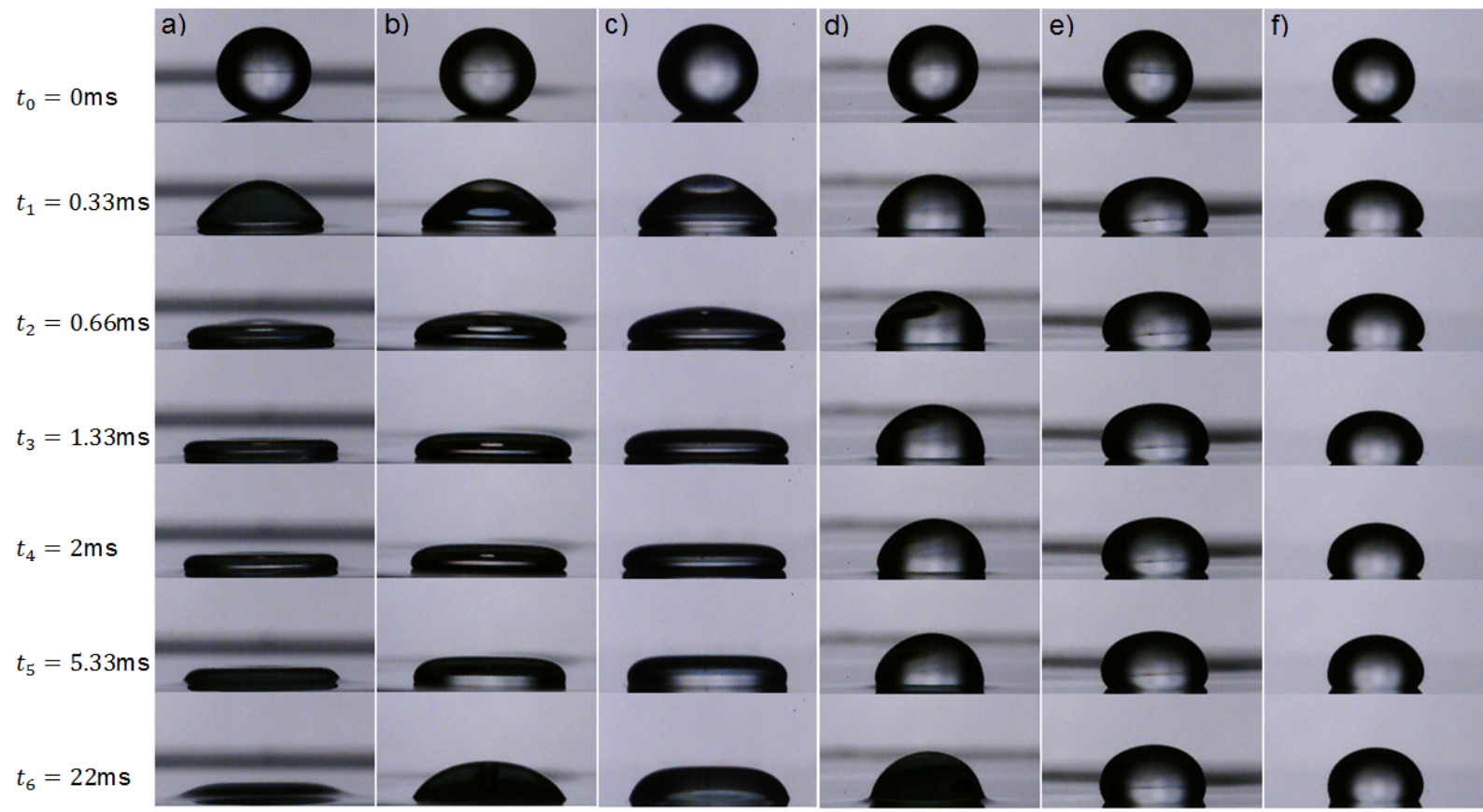

Figure 3. Temporal development of the drop spreading of a $S 350$ drop (a)-c)) and a $S 10000$ drop (d)-f)) impacting onto different impact substrates, wall film of $S 1000(a), d)$ ) and of $S 100000(b), e)$ ) with the same relative film thickness $\delta=0.227$ and glass (c), f)).

it can also lead to corona splash depending on the impact substrate. The impact outcome itself is influenced by the viscosity of the wall film whereby the maximum spreading diameter $D_{\max }$ and maximum corona diameter $D_{c r, \max }$ are highly influenced by the viscosity of the drop as it can be also observed in the case of deposition. It can be observed that with increasing viscosity of the drop $D_{c r, \max }$ decreases whereas the increase of the viscosity of the wall film does not significantly influence $D_{c r, \max }$ as shown in Fig. 4.

The fluid of the drop seems to predominate the impact outcome. In the case of splashing though it can be observed that the fluid of the wall film determines the splashing threshold as it can be seen in Fig. 4. In the case of a highly viscous drop the splashing threshold is already reached at a wall film viscosity of $\nu_{f}=20 \mathrm{~mm}^{2} / \mathrm{s}$.

The impact substrate also determines the impact outcome. For a high viscous wall film the drop cannot evolve to a corona since the resistance of the wall film is too high and does not admit the evolution of a corona. The drop can only slightly penetrate the wall film. Therefore the drop spreads on the high viscous wall film alike on a solid surface, like glass. If the wall film is less viscous the drop impact leads to corona or even to corona splash. In this case the drop is able to penetrate into the wall film, the liquid of the wall film is pushed aside and a corona is built. In both 


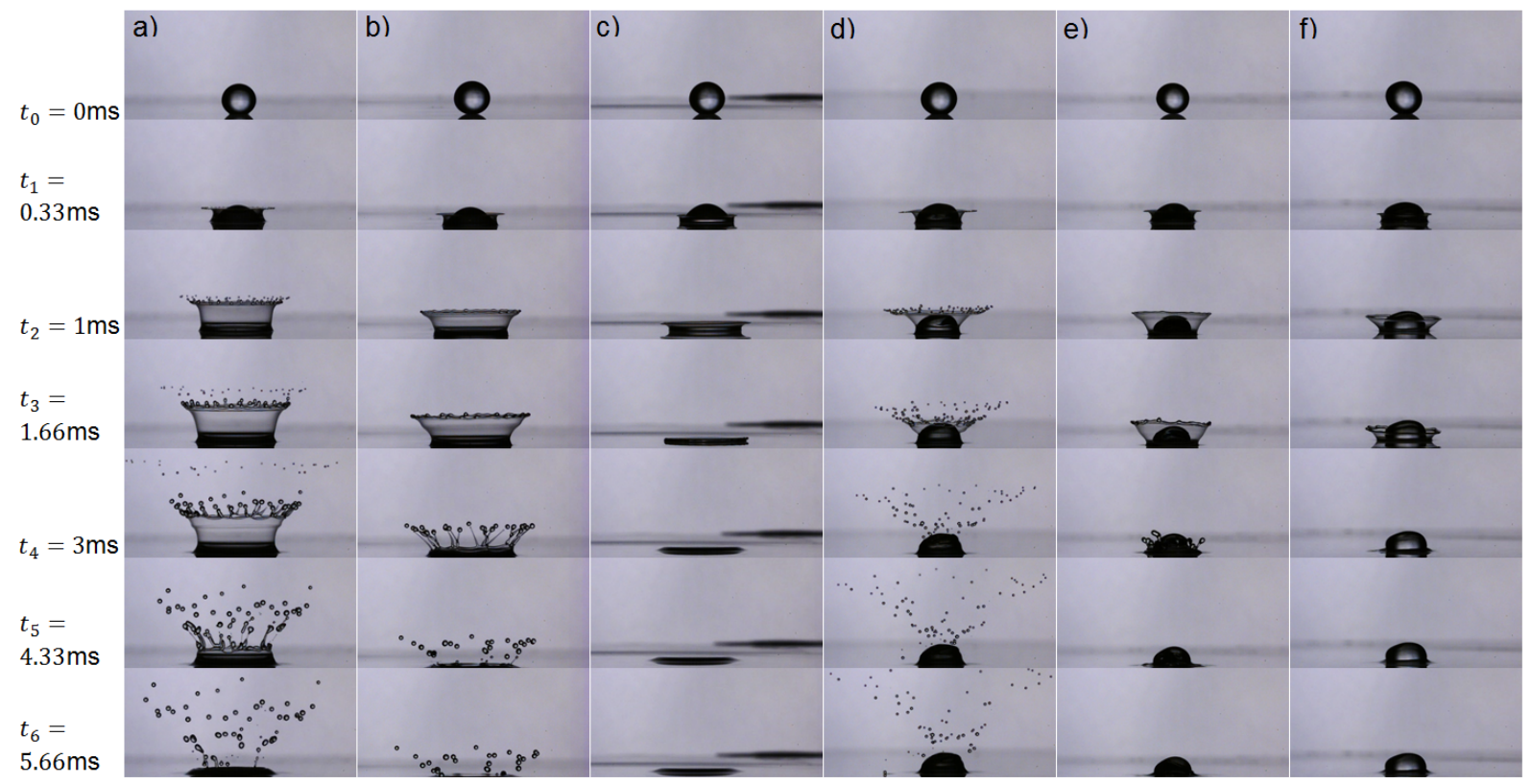

Figure 4. Temporal development of the corona evolution of a $S 350$ drop (a)-c)) and a $S 10000$ drop (d)-f)) impacting onto different impact substrates, wall film of S5 (a), d)), S10 (b), e)) and S20 (c), f)) for the same relative film thickness $\delta=0.045$.

cases the maximum spreading diameter and the maximum corona diameter vary dependent on the viscosity of the drop. Similar to a high viscous wall film a high viscous drop does not change its shape too much. In contrast a less viscous drop extends into the wall film.

In order to determine the effect of a high viscosity liquid on the process of spreading the dimensionless maximum spreading diameter $D_{\max } / D_{0}$ and the dimensionless maximum spreading time $\tau$ are evaluated. The dimensionless maximum spreading time $\tau$ is defined below in Eq.1,

$$
\tau=t_{\max } \frac{u_{0}}{d_{0}} R e_{d}^{-1 / 5}
$$

with the impact velocity $u_{0}$, the initial drop diameter $d_{0}$ and the Reynolds number of the drop

$$
R e_{d}=\frac{u_{0} d_{0}}{\nu_{d}}
$$

The indices $d$ and $f$ represent $d r o p$ and $f i l m$, respectively. $\frac{u_{0}}{d_{0}} R e^{-1 / 5}$ is the typical time of expansion of the boundary in the drop [9].

In Fig. 5a) the dimensionless maximum spreading time $\tau$ is shown as a function of the relative film thickness $\delta$ and the viscosity ratio $\nu_{0}$. For a range of viscosities the value of $\tau$ is near to unity. Therefore the time of drop deformation is determined by the expansion of the boundary layer. It can be seen that with increasing viscosity of the drop impacting drop onto different high viscous liquids the dimensionless time $\tau$ decreases respectively its dependence on $\delta \nu_{0}^{1 / 6}$. However, the dependency of $\tau$ on $\delta$ is higher than the dependency on $\nu_{0}$ what is expressed by $\delta \nu_{0}^{1 / 6}$. The dependency of the dimensionless time $t_{\max } u_{0} / d_{0}$ as a function of the Reynolds number of the drop on the viscosity of the drop in the case of glass as impact substrate is shown in Fig. 5b). In the transition region of low and high viscous liquids expressed by the Reynolds number a jump can be seen. For $R e_{d}<20$ the dimensionless spreading time is not dependent on $R e_{d}$ anymore. The gap of the transition region seems to occur due to the characteristic of the low viscous and high viscous liquids to move very fast and accordingly to stop its motion very fast what leads to comparable dimensionless spreading times in the case of glass as impact substrate.

The effect of the viscosity of the wall film on the dimensionless maximum spreading diameter is shown in Fig. 6a). A clear correlation between $D_{\max } / d_{0}$ and $R e_{d}$ can be seen for the different impact substrates. Furthermore all results are rather in line with each other. This shows that the drop impact onto a high viscous wall film can be compared to the drop impact onto a glass substrate. The experimental results are compared with a model of the maximum spreading diameter by Roisman [9] and is valid for high Reynolds numbers.

$$
D_{\text {max }} \approx 0.87 R e^{1 / 5}-0.4 R e^{2 / 5} W e^{1 / 2} .
$$

The theoretical $D_{\max }$ matches the experimental data rather well. For $R e \geq 0$ the theoretical $D_{\max }$ and the experimental $D_{\max } / d_{0}$ differ from each other.

If the fluid of the wall film is less viscous, but the fluid of the drop is high viscous, a corona is evolved. The maximum corona diameter $D_{c r, \max }$ is in this connection highly dependent on the viscosity of the drop as it can be seen in Fig. 6b). Since all results are rather in line with each other the viscosity of the wall film does not seem to influence 


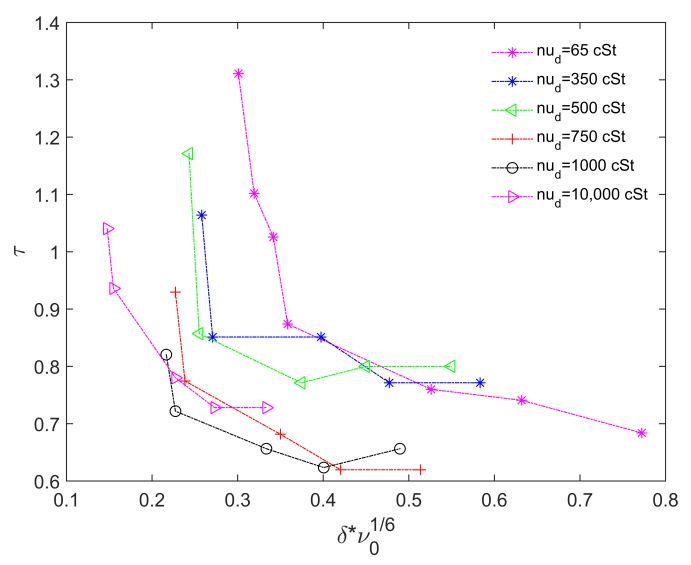

(a)

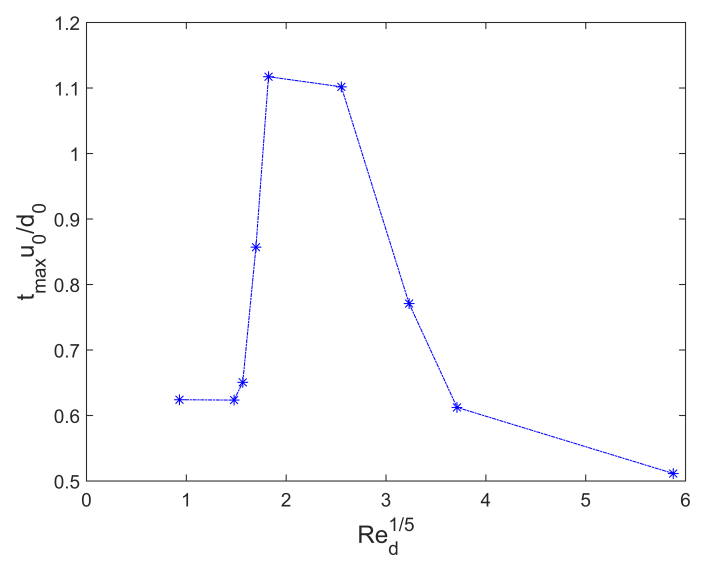

(b)

Figure 5. a) $\tau$ defined in Eq. (1) as a function of the relative film thickness $\delta$ and the viscosity ratio $\nu_{0}$ and of b) the dimensionless spreading time $t_{\max } u_{0} / d_{0}$ as a function of the Reynolds number of the drop $R e_{d}$ for a drop impacting onto glass.

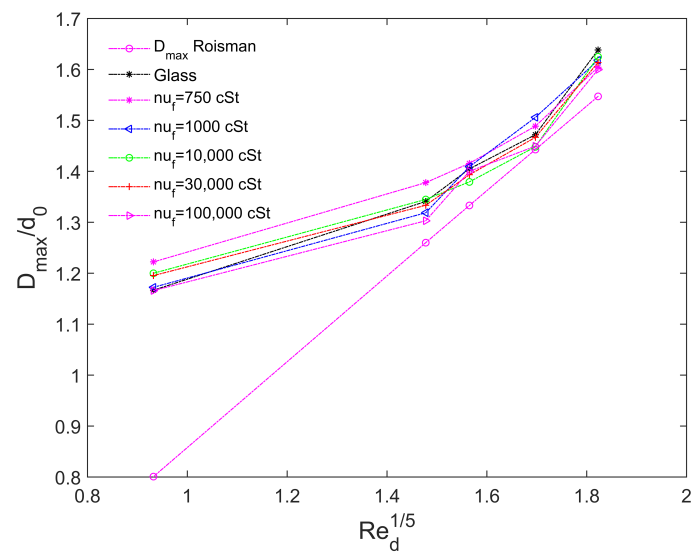

(a)

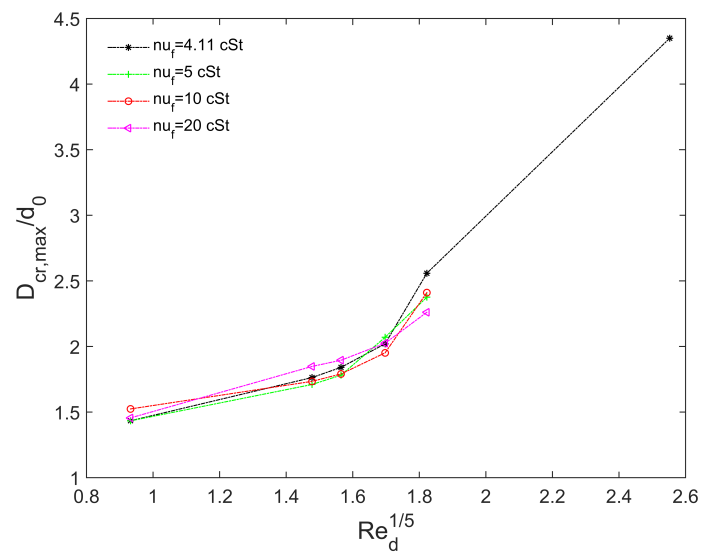

(b)

Figure 6. a) Dimensionless maximum spreading diameter as a function of the Reynolds number of the drop $R e_{d}$ and b) Dimensionless maximum corona diameter as a function of the Reynolds number of the drop $R e_{d}$ for the relative film thickness $\delta=0.045 \mathrm{~mm}$.

$D_{c r, \max }$ significantly. Solely the impact outcome itself, corona or corona splash, is influenced by the viscosity of the wall film as shown in Fig. 4. A high viscous drop does not tend to spread after the drop impact what can already be seen in Fig. 3 in the case of deposition. Due to its contour accuracy which comes from the high viscosity the drop only pushes the liquid of the wall film aside but does not extend itself. Due to the kinetic energy resulting from the impact the shape of the corona is determined by the film liquid which is pushed aside while the drop nearly keeps its initial form. Therefore the maximum corona diameter decreases with decreasing Reynolds number.

If splashing occurs, it is desirable to define a splashing threshold. The dimensionless $\mathrm{K}$ number has been introduced in order to define a critical value to determine the splashing threshold in the case of a one component system [13] as

$$
K=W e R e^{1 / 2}
$$

with the Weber number

$$
W e=\frac{u_{0}^{2} d_{0} \rho}{\sigma}
$$

with the density $\rho$ and the surface tension $\sigma$ and the Reynolds number as described in Eq.2.

If the liquids of droplet and wall film are different, the modified $\mathrm{K}^{*}$ number is used as introduced in [15] since both fluid properties influence the impact outcome. The modified $\mathrm{K}^{*}$ number is defined as

$$
K^{*}=\frac{d^{3 / 2} \rho u^{5 / 2}}{\sqrt{\bar{\nu}} \sigma}
$$


with

$$
\begin{aligned}
& \bar{\nu}=\frac{\nu_{f}^{3 / 2}+\nu_{d}^{3 / 2}}{\nu_{f}^{1 / 2}+\nu_{d}^{1 / 2}}, \\
& \bar{\sigma}=\frac{\sigma_{f}+\sigma_{d}}{2}
\end{aligned}
$$

and

$$
\bar{\rho}=\frac{\rho_{f}+\rho_{d}}{2} .
$$

The effective viscosity is estimated on the assumption that the average film thickness in the corona is scaled by the

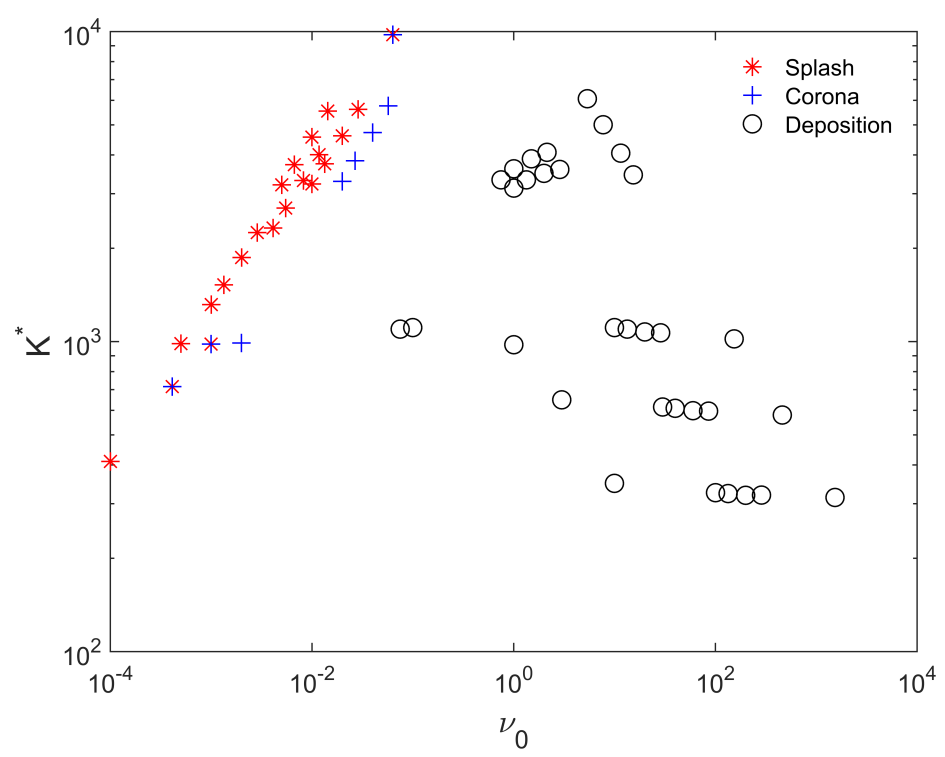

Figure 7. Impact outcome illustrated by the $\mathrm{K}^{*}$ number as function of the viscosity ratio $\nu_{0}$.

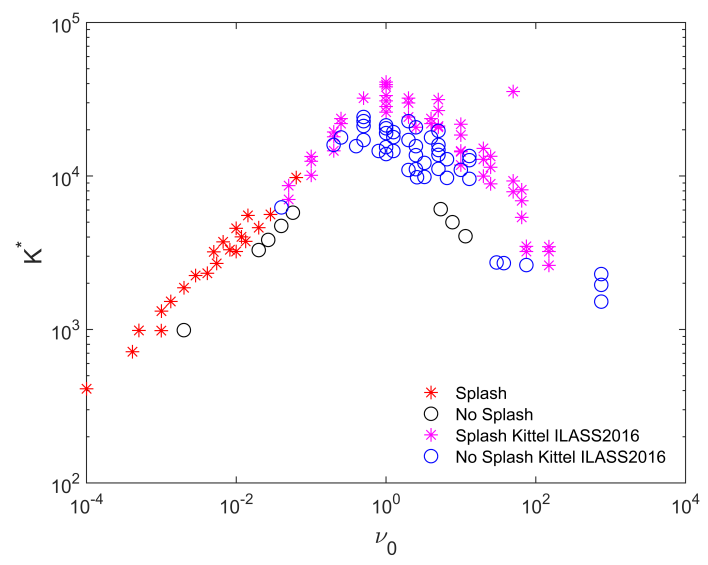

(a)

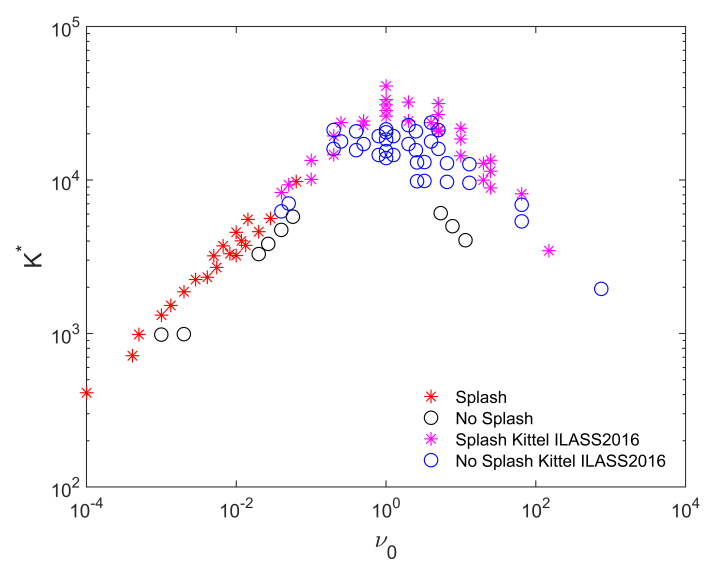

(b)

Figure 8. The modified $\mathrm{K}^{*}$ number as a function of the viscosity ratio $\nu_{0}$ compared to the results from Kittel [15] for the film thickness a) $h=0.1 \mathrm{~mm}$ and b) $h=0.25 \mathrm{~mm}$.

thickness of the viscous boundary layer $\sqrt{\nu t}$. The average viscosity in the composed film of both liquids is estimated as the film thickness average.

In Fig. 7 the $\mathrm{K}^{*}$ number is shown as a function of the viscosity ratio $\nu_{0}$ for the film thicknesses used in this study. A clear splashing/corona limit can be seen. More experimental data is needed to sharpen the corona/deposition limit, a fist tendency can be seen so far. A transition of splashing to corona and finally to deposition can be seen. The in 
this study observed phenomena,deposition, corona and corona splash, can be differentiated using the modified $\mathrm{K}^{*}$ number as a first approximation. It has to be noted that more experimental data is needed.

In Fig. 8 the results relating to the film thickness $h=0.1 \mathrm{~mm}$ in a) and $h=0.25 \mathrm{~mm}$ in b) are compared to the results of Kittel et al. [15]. The results of this study for both film thicknesses fit the data of Kittel et al. [15] very well. The correlation between the modified $\mathrm{K}^{*}$ number and the viscosity ratio $\nu_{0}$ seems to work for numerous liquid combinations, like low viscous - low viscous or low viscous - high viscous.

\section{Conclusions}

In this study the effect of a high viscous drop impacting onto a wetted wall on the impact outcome is investigated. The viscosity of the drop and wall film are varied up to $100,000 \mathrm{~mm}^{2} / \mathrm{s}$ as well as the relative film thickness. The dependency of the maximum spreading diameter in the case of deposition and maximum corona diameter in the case of corona on the Reynolds number is shown. The viscosity of the wall film only influence the impact outcome itself, but the viscosity of the drop determine maximum spreading diameter and maximum corona diameter. In the case of a high viscous drop impacting onto a high viscous wall film the outcome is comparable to the impact on glass except for its final resting shape. The dimensionless maximum spreading time $\tau$ has been introduced to determine the effect of the viscosity of the drop. In the case of a high viscous drop impacting onto a less viscous wall film the maximum corona diameter decreases with increasing drop viscosity. To determine the splashing threshold the modified $K^{*}$ number as a function of the viscosity ratio shows a good correlation.

\section{Acknowledgements}

This research was supported by the the German Scientific Foundation (Deutsche Forschungsgemeinschaft) in the framework of the SFB-TRR 150 collaborative research center, subproject A02.

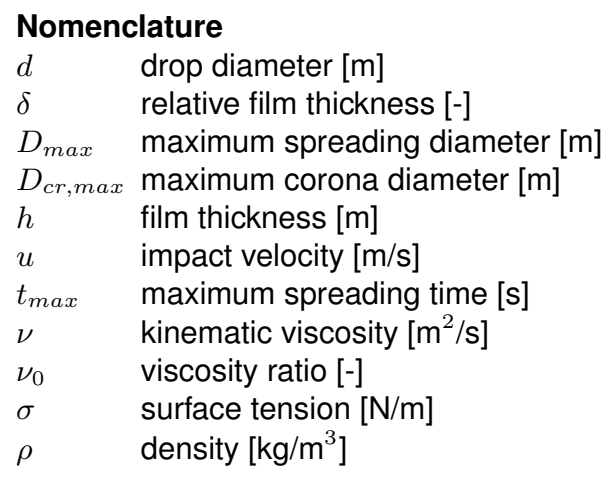

\section{References}

[1] Rein, M., 1993, Fluid Dynamics Research, 12, pp. 61-93.

[2] Yarin, A.L., 2006, Annual Review of Fluid Mechanics, 38, pp. 159-192.

[3] Thoroddsen, S. T., Etoh, T. G., Takehara, K., 2008, Annual Review of Fluid Mechanics, 40, pp. 257-285.

[4] Marengo, M., Antonini, C., Roisman, I. V., Tropea, C., 2011, Current Opinion in Colloid \& Interface Science, 16, pp. 292-302.

[5] Josserand, C. and Thoroddsen, S. T., 2016, Annual Review of Fluid Mechanics, 48, pp. 365-391.

[6] Liang, G., Mudawar, I., 2017, International Journal of Heat and Mass Transfer, 106, pp. 103-126.

[7] Quéré, D., 2013, Annual Review of Fluid Mechanics, 45, pp. 197-215.

[8] Yarin, A. L., Roisman I. V., Tropea, C., 2017, Collision Phenomena in Liquids and Solids, Cambridge University Press.

[9] Roisman, I. V., 2009, Physics of Fluids, 21(5), pp.052104.

[10] Taylor, G., 1959, Proceedings of the Royal Society, 253(1274), pp. 313-321.

[11] Yarin, A.L., Weiss, D.A., 1995, Journal of Fluid Mechanics, 283, pp. 141-173.

[12] Roisman, I.V., Tropea, C., 2006, Journal of Fluid Mechanics, 472, pp. 373-397.

[13] Cossali, G.E., Coghe, A., Marengo, M., 1997, Experimental of Fluids, 22 (6), pp. 463-472.

[14] Roisman, I.V., Horvat, K., Tropea, C., 2006, Physics of Fluids, 18(10), pp. 102-104.

[15] Kittel, H.M., Roisman, I.V. and Tropea, C., Sep. 4.-7. 2016, $27^{\text {th }}$ European Conference on Liquid Atomization and Spray Systems. 